\title{
Comparative genomics of parasitic silkworm microsporidia reveal an association between genome expansion and host adaptation
}

Guoqing Pan ${ }^{1 \dagger}$, Jinshan Xu ${ }^{2,3 \dagger}$, Tian Li ${ }^{1 \dagger}$, Qingyou Xia', Shao-Lun Liu ${ }^{3}$, Guojie Zhang ${ }^{4}$, Songgang Li ${ }^{4}$, Chunfeng Li ${ }^{1}$, Handeng Liu', Liu Yang ${ }^{1}$, Tie Liu', Xi Zhang ${ }^{2}$, Zhengli Wu', Wei Fan ${ }^{4}$, Xiaoqun Dang ${ }^{1}$, Heng Xiang ${ }^{1}$, Meilin Tao ${ }^{1}$,

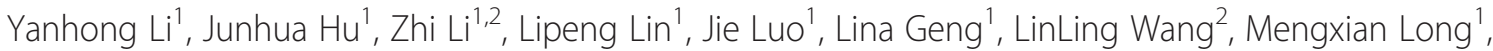
Yongji Wan', Ningjia He', Ze Zhang ${ }^{1}$, Cheng Lu', Patrick J Keeling ${ }^{3}$, Jun Wang ${ }^{4}$, Zhonghuai Xiang ${ }^{1}$ and Zeyang Zhou ${ }^{1,2^{*}}$

\begin{abstract}
Background: Microsporidian Nosema bombycis has received much attention because the pébrine disease of domesticated silkworms results in great economic losses in the silkworm industry. So far, no effective treatment could be found for pébrine. Compared to other known Nosema parasites, N. bombycis can unusually parasitize a broad range of hosts. To gain some insights into the underlying genetic mechanism of pathological ability and host range expansion in this parasite, a comparative genomic approach is conducted. The genome of two Nosema parasites, N. bombycis and N. antheraeae (an obligatory parasite to undomesticated silkworms Antheraea pernyi), were sequenced and compared with their distantly related species, N. ceranae (an obligatory parasite to honey bees).

Results: Our comparative genomics analysis show that the N. bombycis genome has greatly expanded due to the following three molecular mechanisms: 1) the proliferation of host-derived transposable elements, 2) the acquisition of many horizontally transferred genes from bacteria, and 3) the production of abundnant gene duplications. To our knowledge, duplicated genes derived not only from small-scale events (e.g., tandem duplications) but also from largescale events (e.g., segmental duplications) have never been seen so abundant in any reported microsporidia genomes. Our relative dating analysis further indicated that these duplication events have arisen recently over very short evolutionary time. Furthermore, several duplicated genes involving in the cytotoxic metabolic pathway were found to undergo positive selection, suggestive of the role of duplicated genes on the adaptive evolution of pathogenic ability.

Conclusions: Genome expansion is rarely considered as the evolutionary outcome acting on those highly reduced and compact parasitic microsporidian genomes. This study, for the first time, demonstrates that the parasitic genomes can expand, instead of shrink, through several common molecular mechanisms such as gene duplication, horizontal gene transfer, and transposable element expansion. We also showed that the duplicated genes can serve as raw materials for evolutionary innovations possibly contributing to the increase of pathologenic ability. Based on our research, we propose that duplicated genes of $\mathrm{N}$. bombycis should be treated as primary targets for treatment designs against pébrine.
\end{abstract}

Keywords: Gene duplication, Horizontal gene transfer, Host-derived transposable element, Host adaptation, Microsporidian, Silkworms

\footnotetext{
* Correspondence: zyzhou@swu.edu.cn

${ }^{\dagger}$ Equal contributors

'State Key Laboratory of Silkworm Genome Biology, Southwest University,

Chongqing 400715, China

${ }^{2}$ College of Life Sciences, Chongqing Normal University, Chongqing 400047,

China

Full list of author information is available at the end of the article
}

\section{Biomed Central}

(C) 2013 Pan et al.; licensee BioMed Central Ltd. This is an Open Access article distributed under the terms of the Creative Commons Attribution License (http://creativecommons.org/licenses/by/2.0), which permits unrestricted use, distribution, and reproduction in any medium, provided the original work is properly cited. 


\section{Background}

Microsporidia are obligate intracellular parasitic fungi that can infect a wide variety of organisms including vertebrate and invertebrate (particularly insects). Some species lead to severe syndromes in immunocompetent hosts and cause opportunistic infections in Acquired Immunodeficiency Syndrome (AIDS) patients [1,2]. More than 1200 microsporidia species that belong to 150 genera have been reported thus far [3]. Among them, the genus Nosema is the most diverse one. The domesticated silkworm, Bombyx mori, has long been considered as the primary source for the silk production worldwide. A highly mortal disease referred to as pébrine is currently the major threat to the silk production. Pébrine is caused by the infection of the microsporidian parasite, Nosema bombycis. This disease was first recognized during the destruction of the European silk industry in 1857 [4]. N. bombycis infects silkworms through vertical transmission from the mother host to their progenitive eggs, and chronically damages the entire body of the worm (including intestines, silk glands, muscles, and Malpighian tubules). After infections, the silkworm larvae are inactive and slow in development. Later, black spots, a disease symptom called pébrine [5], will appear throughout their bodies and lead to death. Since no effective treatment methods have been developed up to this point, the infections by $N$. bombycis inevitably cause devastating economic losses in the silkworm industry. Apart from the domesticated silkworms, N. bombycis can also infect various lepidopteran insects [6-8], indicative of their broad hosts range.

So far, the underlying genetic mechanisms of the highly infectious ability and the broad host range of $N$. bombycis remain unknown. To this end, we conducted a comparative genomic approach, from which we might learn a great deal about the genetic basis as to why and how $N$. bombycis can be so infectious across various hosts. In this study, we sequenced the genome of two microsporidian parasites: $N$. bombycis and $N$. antheraeae (an obligatory parasite to undomesticated silkworms Antheraea pernyi [9]). By comparing their genomes with a published distantly related Nosema genome, N. ceranae [10] (serving as outgroup), we show that the $N$. bombycis genome surprisingly expands due to the production of duplicated genes, the proliferation of host-derived transposable elements, and the acquisitions of many horizontally transferred genes from bacteria. Some duplicated genes associated with the cytotoxic pathway have experienced positive selection, implying that this adaptive evolution might enhance the infectious ability of $N$. bombycis, as well as the expansion of its host range. Considering that all reported microsporidian genomes are highly reduced and compact $[11,12]$, our data, for the first time, reveal a usual genome evolution process showing that the genome of parasites could expand. Those expanded genetic gears might have influenced the infectivity and the survivorship of parasites as we report herein.

\section{Results}

Genomic architecture of $\mathrm{N}$. bombycis and $\mathrm{N}$. antheraeae By using various sequencing platforms, 6.7X, 10X, and $28 \mathrm{X}$ physical coverage of whole genome sequence of $N$. bombycis were obtained from the Sanger sequencing method (plasmids with $2 \mathrm{~Kb}$ inserts), the miniBAC end sequencing method, and the Illumina short-read sequencing method respectively (Additional file 1). Our sequencing efforts resulted in 1,605 scaffolds built from 3,551 contigs. The total assembly genome size is $15.7 \mathrm{Mb}$ (the $\mathrm{N} 50$ of the scaffolds $=57.4 \mathrm{~Kb}$ and the maximum scaffold size $=571.1 \mathrm{~Kb})($ Additional file 2). A total of 4,458 protein coding sequences were identified (Table 1). The assembled genomic size $(\sim 15.7 \mathrm{Mb})$ is close to previous estimation by pulse-field gel electrophoresis $(\sim 15.3 \mathrm{Mb})$ [13], indicating that the coverage of the assembled genome is nearly complete. To sequence the genome of $N$. antheraeae, a total of $657 \mathrm{Mb}$ of Illumina reads were obtained after filtering ambiguous reads. Our analysis results in 6,215 scaffolds and a total of 6.6 Mb of unique sequence. Totally, 3,413 protein coding sequences were identified (Table 1). The assembled genome size of $N$. antheraeae was estimated using the following equation: genome size = number of 15-mers per kilo-bases/depth of 15-mers per kilo-bases. The assembled genome size of $N$. antheraeae $(\sim 7.4 \mathrm{Mb})$ (Additional file 3 ) is close to our previous estimation by pulse-field gel electrophoresis [14], indicating that approximately $90 \%$ of the N.antheraeae genome was captured. A comparison of genome features among the generated Nosema genomes and published microsporidian genomes (one Nosema species and two Encephalitozoon species) is listed in Table 1.

To gain some insights into the variations of gene content among $N$. antheraeae, $N$. bombycis, and $N$. ceranae, the number of orthologous genes were compared. Most genes are shared among all three species, but $8 \%$ of genes were $N$. antheraeae-specific, $15.7 \%$ were $N$. bombycis-specific, and $30.5 \%$ were $N$. ceranae-specific (Figure 1). Gene ontology analysis revealed that no distinct differences were found among different gene functional categories (Additional file 4). Collectively, these three Nosema species lack genes for tricarboxylic acid cycle, oxidative phosphorylation, and fatty acid $\beta$-oxidation, consistent with previous observations [15-19]. Our observations further support that microsporidia do not possess tricarboxylic acid cycle and oxidative metabolism, and microsporidia parasites have often experienced on-going genome streamlining via the relaxation of purifying selection.

To determine if the Nosema proteins were more compact than other microsporidian parasites, the length of those silkworm Nosema proteins with assigned functions 
Table 1 A comparison of genome features among three Nosema species (N. bombycis, N. antheraeae, and N. ceranae) and two Encephalitozoon species (E. cuniculi and E. bieneusi)

\begin{tabular}{llllll}
\hline Genomic features & N. bombycis & N. antheraeae & N. ceranae [10] & E. cuniculi [24] & E. bieneusi [26] \\
\hline Chromosomes(bands) & 18 & $\sim 12$ & ND & 11 & 6 \\
Assembly(Mbp) & 15.7 & 6.6 & 7.9 & 2.9 & 3.86 \\
Genomic coverage & $100 \%$ & $\sim 89 \%$ & $90 \%$ & $86 \%$ & $\sim 64 \%$ \\
Scaffold Num & 1,605 & 6,215 & 5,465 & 11 & 1,646 \\
N50(bp) & 57,394 & 1,883 & 2,902 & ND & 2,349 \\
Largest scaffold length(bp) & 571,060 & 53,183 & 65,607 & 209,983 & 204,069 \\
G + C content (\%) & 31 & 28 & 27 & 48 & 26 \\
No .of CDS & 4,458 & 3,413 & 2,614 & 1,997 & 3,632 \\
Mean CDS length (bp) & 741 & 775 & 904 & 1,077 & NA155 \\
GenBank No. & NA30919 & NA183977 & NA32971 & NA21011 \\
\hline
\end{tabular}

was compared to homologs of two published microsporidian parasitic Encephalitozoon species, E. cuniculi and E.intestinalis (Additional file 5). Our results show that the average length of total homologous genes from $N$. antheraeae and $N$. bombycis is shorter than that from E. cuniculi and E. intestinalis, indicating that proteins in Nosema were more compact than those in Encephalitozoon.

Overall, our comparative genomics analysis showed that $N$. bombycis possesses a much larger genome size than other two Nosema species (Table 1). Considering that $N$. bombycis has wide host range, the genome expansion might facilitate the host adaption in $N$. bombycis. Thus, for the subsequent analyses, we aim to seek for the underlying genetic mechanisms as to why and how $N$. bombycis genome expands. Furthermore, we seek for the putative genetic components that contribute to the infectious ability of $N$. bombycis in a hope that our analyses could provide some clues on the development of treatment strategies of pébrine.

\section{Proliferation of host-derived transposable elements in N. bombycis}

After obtaining the genomes of the two Nosema species, we seek for the potential molecular mechanisms underlying the genome expansion of $N$. bombycis. Considering that the proliferation of transposable elements often contributes to the genome size variation in many eukaryotes [20], it was considered as the first molecular mechanisms for us to check. Although the genomes of several human pathogenic microsporidians have been shown to lack transposable elements, transposable elements have been detected in the genomes of other non-human pathogenic microsporidians [21-25]. To understand what degree those transposable element shape the genomic architectures in Nosema, we searched for transposons in N. bombycis and $N$. antheraeae (for details, see Materials and Methods). Two different approaches were implemented in this study.
Because most transposable elements comprise internal protein-coding genes (e.g., transposase or reverse transcriptase) that are necessary for their transposition, we first identified those putative transposable elements by searching for their internal protein-coding sequences. In many cases, the internal protein-coding sequences are highly generated but recognizable. Second, for those that do not possess readily identifiable internal protein-coding sequences, other features such as inverted repeats or insertion sites were used to recognize the transposable elements.

Overall, our results show evidence that a larger genome size of $N$. bombycis is partly due to both the acquisition of new transposons and expansion of existing transposable elements (Table 2). Among all identified transposable elements, the Ty3/gypsy retrotransposons [22] constitute the largest part of known classes of transposable elements in $N$. bombycis. A broad sampling from GenBank shows that these transposable elements also reside in other microsporidian groups including Spraguea lophii, Edhazardia aedis, and Brachiola algerae, indicating that this transposable element family exists back to the common ancestor of most microsporidian species and further expand in N. bombycis. Majority of transposable elements among Nosema genomes are common across three Nosema species, whereas Piggybac transposons were only found in $N$. antheraeae and $N$. bombycis except for $N$. ceranae (Additional file 6). To test whether Piggybac was lost during the evolution of $N$. ceranae or was gained in the most recent common ancestor of $N$. antheraeae and $N$. bombycis, the phylogeny of Piggybac was reconstructed from Nosema, domesticated silkworms, and other insects. Our analyses show that the Nosema Piggybac sequences fall into four well-supported groups, and three out of them are closely related to Piggybac elements from domesticated silkworms (Figure 2). Although the exact relationships of these Piggybac elements between Nosema and Bombyx is complicated, our 


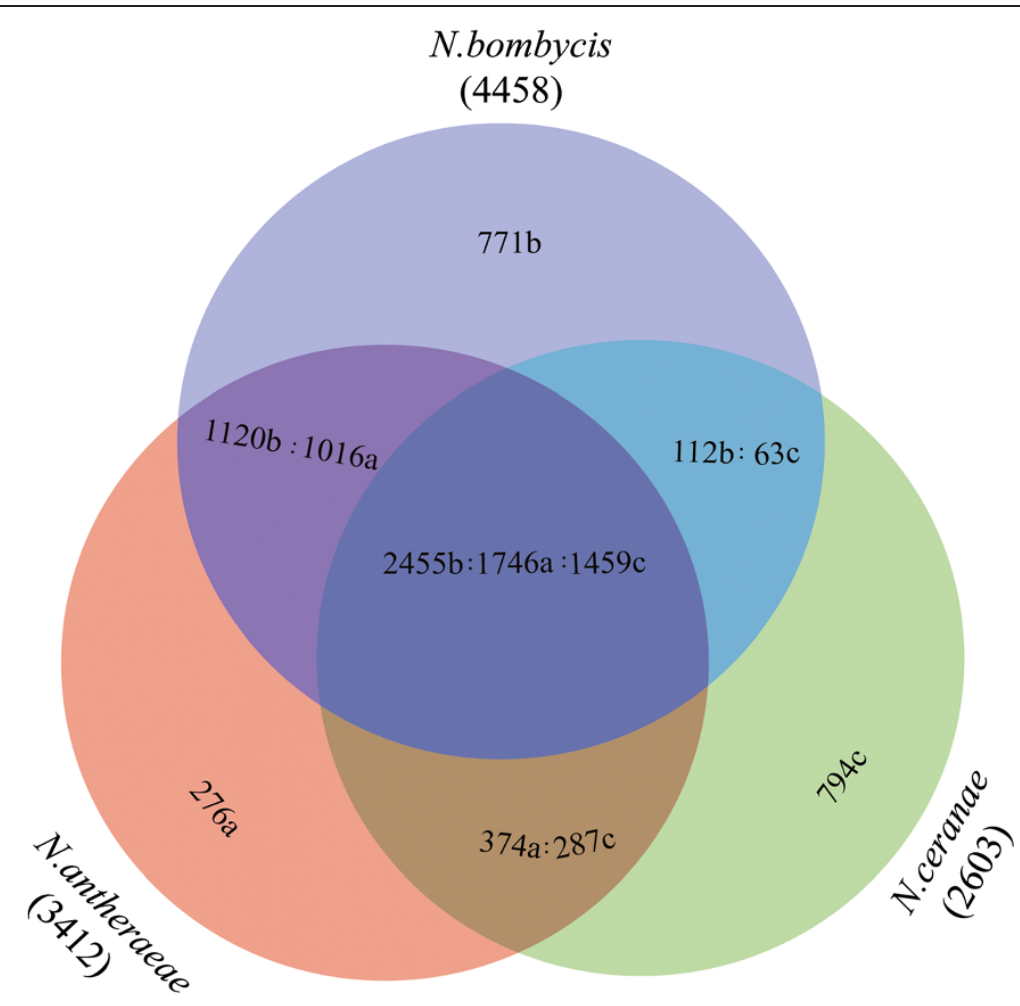

Figure 1 Venn diagram showing the number of homologous genes and lineage-specific genes amongst three Nosema species,

$\boldsymbol{N}$. bombycis, $\boldsymbol{N}$. antheraeae, and $\boldsymbol{N}$. ceranae. The arabic numbers followed by characters represent the number of homologous genes in each Nosema species ('a' denotes $N$. antheraeae, 'b' denotes $N$. bombycis, and ' $c$ ' denotes $N$. ceranae). For instance, 2455b:1746a:1459c means that 2455 genes of $N$. bombycis, 1746 genes of $N$. antheraeae, and 1459 genes of $N$. ceranae are homologous to each other.

phylogenetic analysis suggests that Piggybac was acquired in the most common ancestor of $N$. antheraeae and $N$. bombycis through horizontal transfer events from possibly host silkworms. These transfers likely independently took place three times, leading to the three major subgroups (labeled as HGT in Figure 2). In addition, the Nosema $p B a c 3,4,5$ are closely related to the elements from other insects (in the middle part of tree; Figure 2), suggesting that these Nosema Piggybac elements might originate from the insects. To rule out the possibility of the host contaminations during the genome assembly, we amplified regions flanking Piggybac elements using Nosema-specific primers, and confirmed the existence of Piggybac in Nosema genomes (data not shown).

Since the host-derived Piggybac elements are so abundant in $N$. bombycis, can those host-derived Piggybac elements serve as the vector of capturing host-derived genes? To answer this question, we checked the hostderived Piggybac elements that do not have any readily identifiable internal protein-coding sequences. Because they are usually hard to be identified due to the lack of the internal readily recognizable protein-coding genes, the terminal inverted repeat (ITR) and the insertion site
Table 2 Classification of repetitive families in $\mathbf{N}$. bombycis genome

\begin{tabular}{llll}
\hline Type & Subtype & Length (bp) & Percent (\%) of genome \\
\hline DNA & hAT & $1,011,459$ & 6.45 \\
& Merlin & 470,573 & 3.00 \\
& PiggyBac & 441,876 & 2.82 \\
& TcMar & 786,733 & 5.02 \\
& MuDR & 109,681 & 0.70 \\
& Others & 58,866 & 0.38 \\
LTR & Gypsy & 577,653 & 3.68 \\
& others & 33,635 & 0.21 \\
LINE & Dong-R4 & 162,622 & 1.04 \\
& Others & 59,703 & 0.38 \\
Rolling-circle & Helitron & 102,334 & 0.65 \\
SINE & - & 28,669 & 0.18 \\
Unknown & - & $2,204,497$ & 14.06 \\
Total & - & $6,048,301$ & 38.57 \\
\hline
\end{tabular}


(TTAA) of the Piggybac elements were used as the criteria for our search. In other words, we searched for the $N$. bombycis genomic regions that are flanked by the ITR and the insertion site (TTAA) of the Piggybac elements and comprise "extrinsic" sequences. After identification of those Piggybac elements, we examine whether the "extrinsic" sequences were recently transferred by the transposition of the Piggybac elements that are specific to $N$. bombycis via comparing the colinearity of these regions with those in $N$. antheraeae and N. ceranae. A total of 17 Piggybac elements with an internal "extrinsic" sequence were identified (Additional file 7). Among them, only one case might be recently gained in $N$. bombycis based on the colinearity (Additional file 8 and Additional file 9). When we blasted the internal "extrinsic" sequence of the 17 Piggybac elements in GenBank using the "nr" database by the blastn function, no detectable similarity with any known sequences was found. Our analysis thus far suggests that the host-derived Piggybac elements might not be able to serve as the vector of capturing genes from hosts to $N$. bombycis.

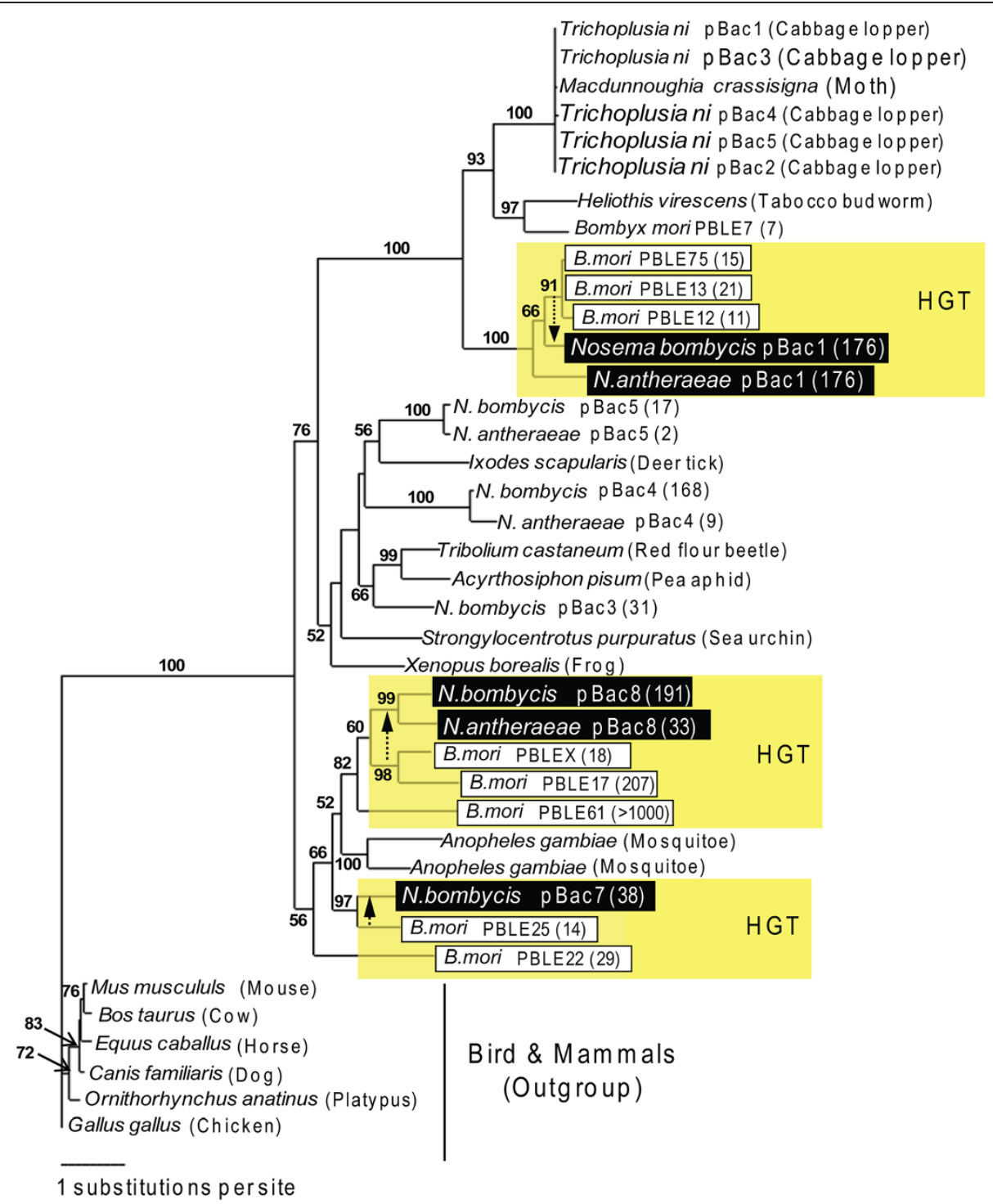

Figure 2 A maximum-likelihood phylogenetic tree of host-derived Piggybac transposase sequences. Arrows show the putative recent horizontal gene transfer (HGT) events of host-derived transposable elements. Several transposons are closely related to those from insects. Black boxes indicate elements from the two silkworm-infecting Nosema species, while white boxes indicate elements from the domesticated silkworm B. mori. Numbers in parentheses indicate the total copy numbers for each transposable element. 


\section{Horizontally transferred protein-coding genes is another source of genetic expansion in $\mathbf{N}$. bombycis}

Since $N$. bombycis has experienced the proliferation of native and host-derived transposons, we sought to determine if horizotally transferred protein coding genes from other organisms can also facilitate the genome expansion in $N$. bombycis, as was recently found in Encephalitozoon [26,27]. To maximize the likelihood of detecting horizontal protein coding gene transfer (HGT) events in $N$. bombycis, we implemented two different approaches: a genome-wide prediction method based on orthologous sequences using the software Darkhorse, and a phylogenetic method where we screened the putative HGTs in a total of 4458 gene family phylogenies. Overall, these two different approaches identify 50 and 53 different HGTs in $N$. bombycis, respectively. Among them, 48 genes are shared between these two approaches (Figure 3A), resulting in a set of 55 union HGT genes between two different dataset. By investigating the taxonomic origin of these 55 unions HGT genes in a phylogenetic framework, all of them were transferred from prokaryotes (Figure 3B). No host-derived genes were found in our analysis, further suggesting that only hostderived transposable elements can be transferred into the $N$. bombycis genome instead of host-derived proteincoding genes. Using the clusters of orthologous group database, we found that 21 HGT candidates are unknown in functions, and 34 are predicted to fall into diverse gene functions (Additional file 10). Among 34 HGT genes, five genes are involved in nucleotide metabolism and two genes are involved in sugar metabolism. Interestingly, one HGT gene that was annotated as phosphomevalonate kinase (EC2.7.4.2) is shown to be an important player in the mevalonate pathway of N.bombycis (Additional file 11). In the mevalonate pathway, phosphomevalonate kinase is a key enzyme to catalyze the rate-limiting step for the production of isopentenyl pyrophosphate (IPP). IPP is important for various molecular functions such as terpenoid synthesis, protein prenylation, cell membrane maintenance, protein anchoring, and N-glycosylation. Overall, our observations lead us to hypothesize that some of HGTs might play a pivotal role on the adaptation or survivorship of $N$. bombycis over the course of evolution. Alternatively, many HGTs might be merely neutral without any immediate adaptive consequences after their transfers. Further hypothesis testing will be necessary.

Recent gene duplication events contribute to the genome expansion in N. bombycis

Although our previous analyses showed that the proliferation of host-derived transposable elements and horizontally transferred genes could contribute to the genome size expansion in $N$. bombycis, their contributions are not sufficient to explain the much larger genome size of
$N$. bombycis than other two small-genome Nosema species (N. antheraeae and N. ceranae). Considering that gene duplication is a common molecular mechanism mediating the expansion of genome size in many eukaryotes [28], we then seek for the evidence if gene duplications also play a role on the genome expansion in $N$. bombycis. We first performed a syntenic analysis to identify possible segmental duplication events in each Nosema species. Among three species, we found that $N$. bombycis contained 942 pairs of segmental duplications throughout its genome (Figure 4A, Additional file 12). In contrast, almost no segmental duplication could be detected in either $N$. antheraeae or $N$. ceranae. Because the assemblies of all these genomes are fragmented, it is not possible to conclude whether these segmental duplications are large in number and spread throughout the genome, or arise due to multiple whole chromosome duplication events or an ancient whole genome duplication event. Nevertheless, we have identified a region where it appears that a single large-scale duplication event explain the data better than several independent large-scale duplication events (Figure 5). To date these duplication events, we estimated synonymous substitution rate $(d S)$ for paralogous genes from segmental duplications in $N$. bombycis, and compared them with the $d S$ derived from orthologs between $N$. antheraeae and $N$. bombycis. The $d S$ values are commonly used as the proxy of age of gene duplication because the synonymous substitutions evolve in a neutral fashion [28]. On average, the $d S$ values of paralogs from segmental duplications in $N$. bombycis are generally lower than that of orthologs between $N$. antheraeae and $N$. bombycis (Figure 4B), suggesting that these duplication events took place after the separation of $N$. antheraeae and $N$. bombycis. In addition to the detection of segmental duplications, we identified numerous tandem duplication events among three Nosema species. We detected a higher rate of tandem duplications in $N$. bombycis compared to other two Nosema species, and in some cases multiple events could be mapped at a single locus (Figure 4C). On average, the $d S$ values of these paralogs are also much lower than that of orthologus genes between $N$. bombycis and $N$. antheraeae (Figure 4D), indicating that most tandem paralogs in $N$. bombycis also arose relatively recent after the separation between $N$. bombycis and $N$. antheraeae. In short, the $N$. bombycis genome has expanded in size largely due to many large-scale and small-scale gene duplication events.

\section{Adaptive evolution of duplicated genes might enhance the pathogenic ability in $\mathrm{N}$. bombycis}

Paralogs often provide raw materials for evolutionary innovations, including the survival of parasites in their hosts [29]. We therefore sought to identify possible instances of adaptive changes associated with the 


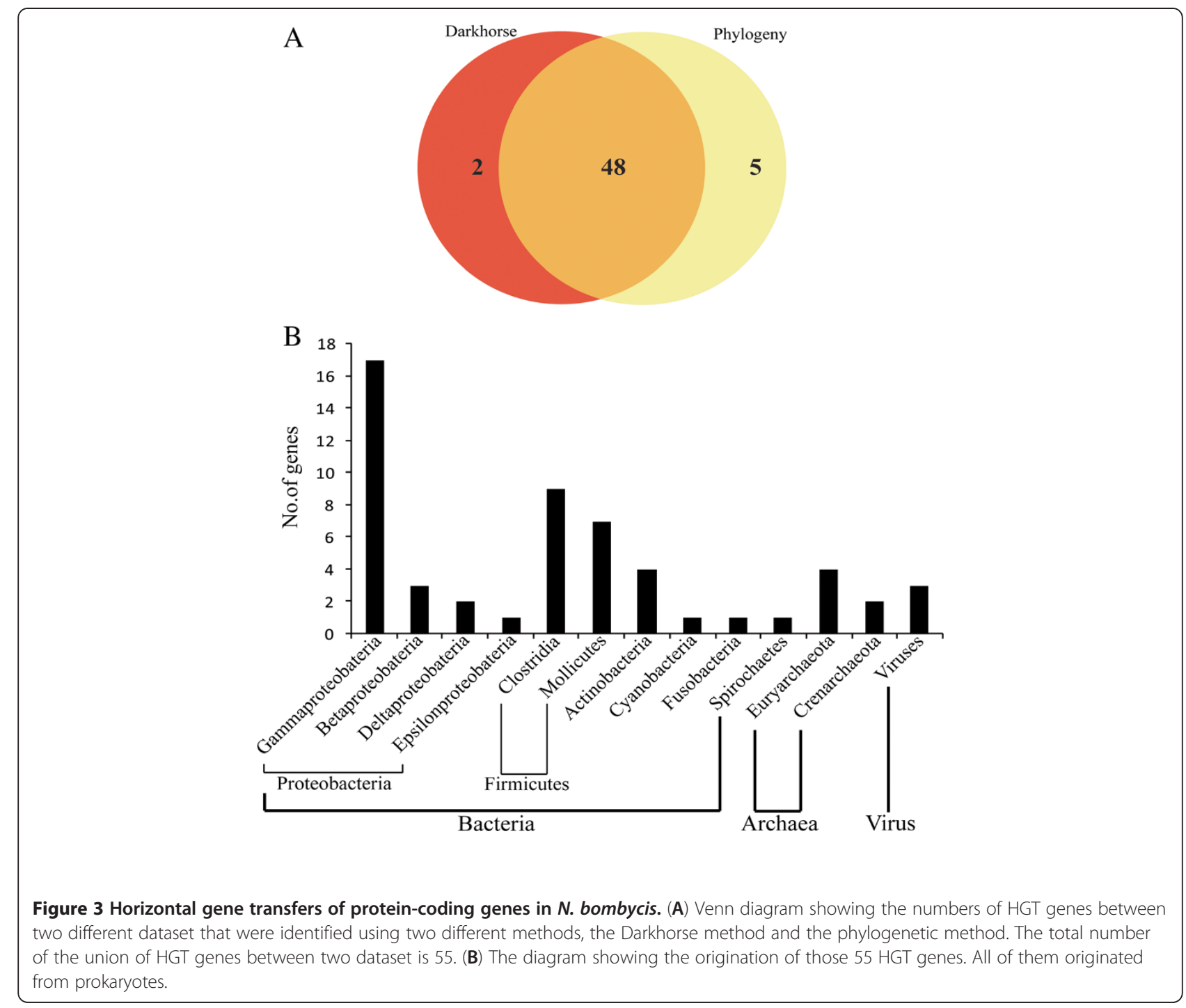

pathogenic ability of $N$. bombycis among those duplicated genes derived from large-scale duplication events in $N$. bombycis. First, we examine if paralogs of $N$. bombycis contribute to the adaptive evolution more often than orthologs among all Nosema species. Clusters of homologous genes in $N$. bombycis were classified to four different groups: 1) clusters of orthologus genes (COGs) of 1:1:1 trios of $N$. bombycis, $N$. antheraeae, and $N$. ceranae, 2) COGs of 1:1 gene pairs of $N$. bombycis and $N$. antheraeae, 3) COGs of 1:1 gene pairs of $N$. bombycis and $N$. ceranae, and 4) clusters of paralogous genes (CPGs) in $N$. bombycis. Pairwise $d N / d S$ ratio analyses for these four different clusters of homologous genes were computed and their cumulative $d N / d S$ ratio curve were compared (see Materials and Methods for details). Compared to COGs, a higher proportion of CPGs in $N$. bombycis showed higher value of $d N / d S$ ratio, suggesting that CPGs are evolving at a faster rate than COGs at the amino acid level (Additional file 13). In most cases, this is likely due to the relaxation of purifying selection. However, we observed that a higher proportion of CPGs showed $d N$ / $d S$ ration greater than 1 , indicative of positive selection. Overall, our observations support the view that CPGs contributed more to adaptive evolution than COGs in $N$. bombycis.

To examine if any particular codons of CPGs of $N$. bombycis have undergone positive selection, we applied a site model approach with maximum likelihood using the software PAML (see Materials and Methods for details). The results show that 24 out of 240 CPGs have experienced positive selection in $N$. bombycis (Table 3 ), and 62\% (37/60) genes in 24 CPGs have the support of EST tags (Additional file 14). The estimated parameters and positively selected sites for those positively selected CPGs are shown in Additional file 15. Although the majority of positively selected CPGs are hypothetical proteins with 

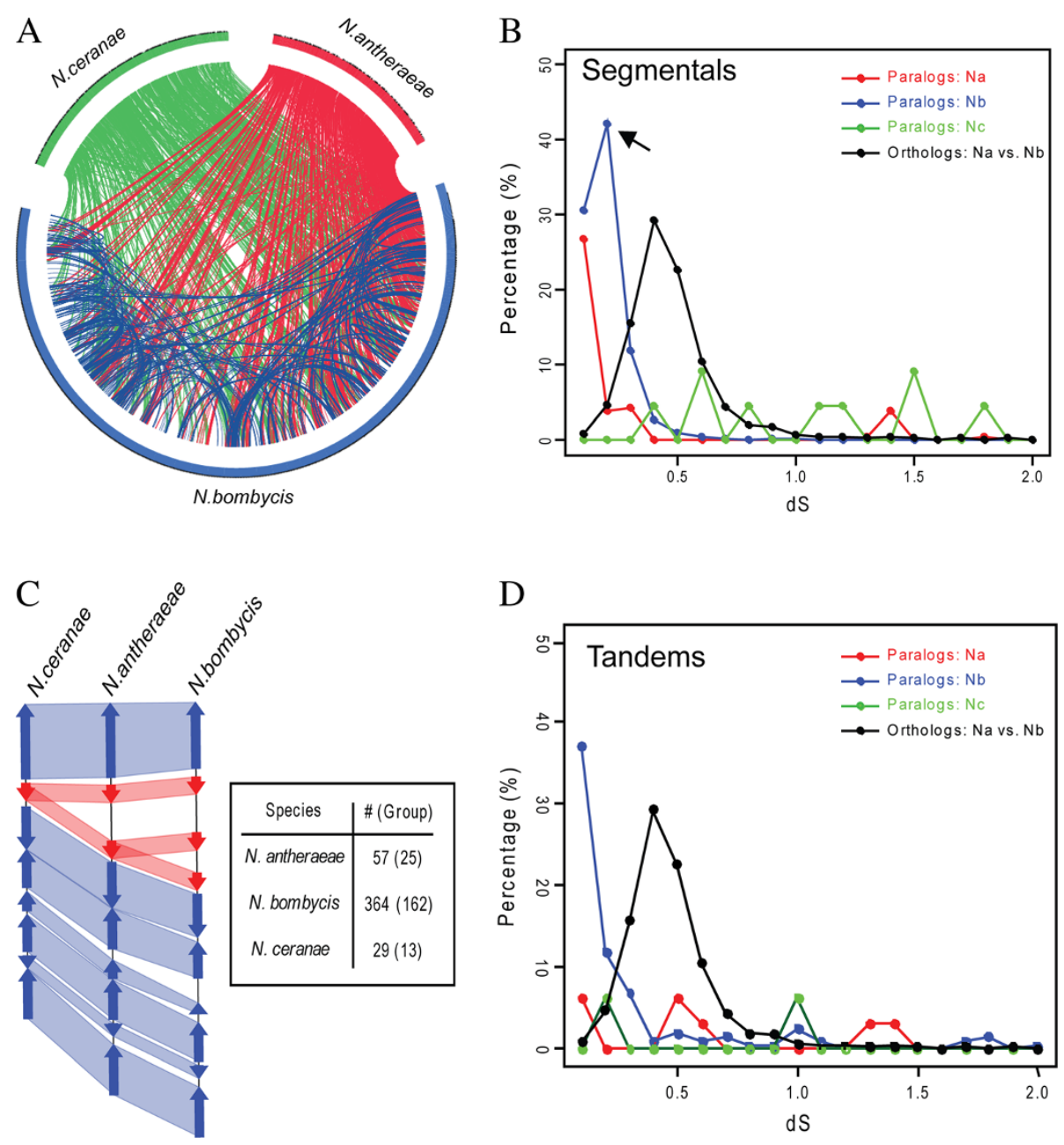

$\mathrm{D}$

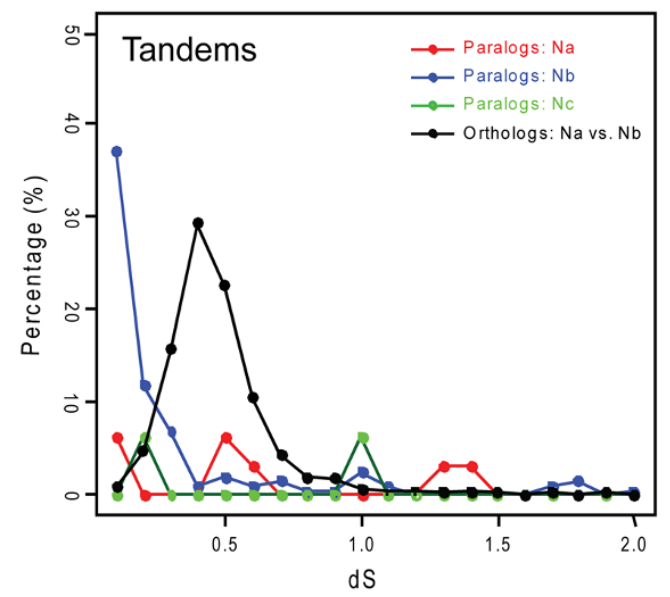

Figure 4 Gene duplications and the dS distribution of paralogs and orthologs among three Nosema species. Abbreviation: Na, N. antheraeae; Nb, N. bombycis; Nc, N. ceranae. (A) A circos map showing the comparative genomics among three different Nosema species based on all available scaffolds. Each line represents the homologous syntenic regions between any two species or between any given two chromosome positions of single species. Many lines across different scaffolds of $\mathrm{N}$. bombysis indicates higher rate of segmental syntenic duplications. (B) The dS distribution of segmental paralogs of $\mathrm{Nb}$ and the orthologs between $\mathrm{Nb}$ and Na showing a higher dS values in orthologs in general. Notably, a higher peak (arrow) seen in Nb suggests the possibility of a burst of paralogs recently over the Nb evolution after the separation of $\mathrm{Na}$ and $\mathrm{Nb}$. (C) An example of syntenic comparisons among three Nosema species showing a cluster of tandem paralogs. The corresponding genetic position and names of identified element are provided in Additional file 8. The number of all identified tandem paralogs for each Nosema genome is summarized on the right side. (D) The dS distribution of tandem paralogs of $\mathrm{Nb}$ and orthologs between $\mathrm{Na}$ and $\mathrm{Nb}$ showing that majorities of tandem paralogs arose after the separation of $\mathrm{Na}$ and $\mathrm{Nb}$ because the $\mathrm{dS}$ values of those tandem paralogs are smaller than that of orthologs.

unknown functions, a handful of them are not. For example, CPG844 is related to LPXTG motif cell wall anchor domain protein and CPG1776 is related to surface adhesion protein. Positive selection acting on these two CPGs might play an important role in host recognition and interaction since they are involved in surface adhesion. Other examples are two positively selected CPGs that are related to serine protease inhibitor (SPN106). The functions of serine protease inhibitor have been shown to decrease the immune responses in hosts
[30-32]. In the melanization pathway of B. mori, serine protease cascade is one of the most important biochemical reactions to inhibit the propagation of pathogens [33]. We scanned the gene expression pattern of B. mori post infection of $N$. bombycis by microarray analysis and found that the key gene PPO in melanization pathway was significantly suppressed. From our gene chip expression analysis (unpublished data), we found that the gene expression of two upstream regulators of host PPO melanization pathway, $\beta-G R P 2$ and $\beta-G R P 4$, in $B$. 


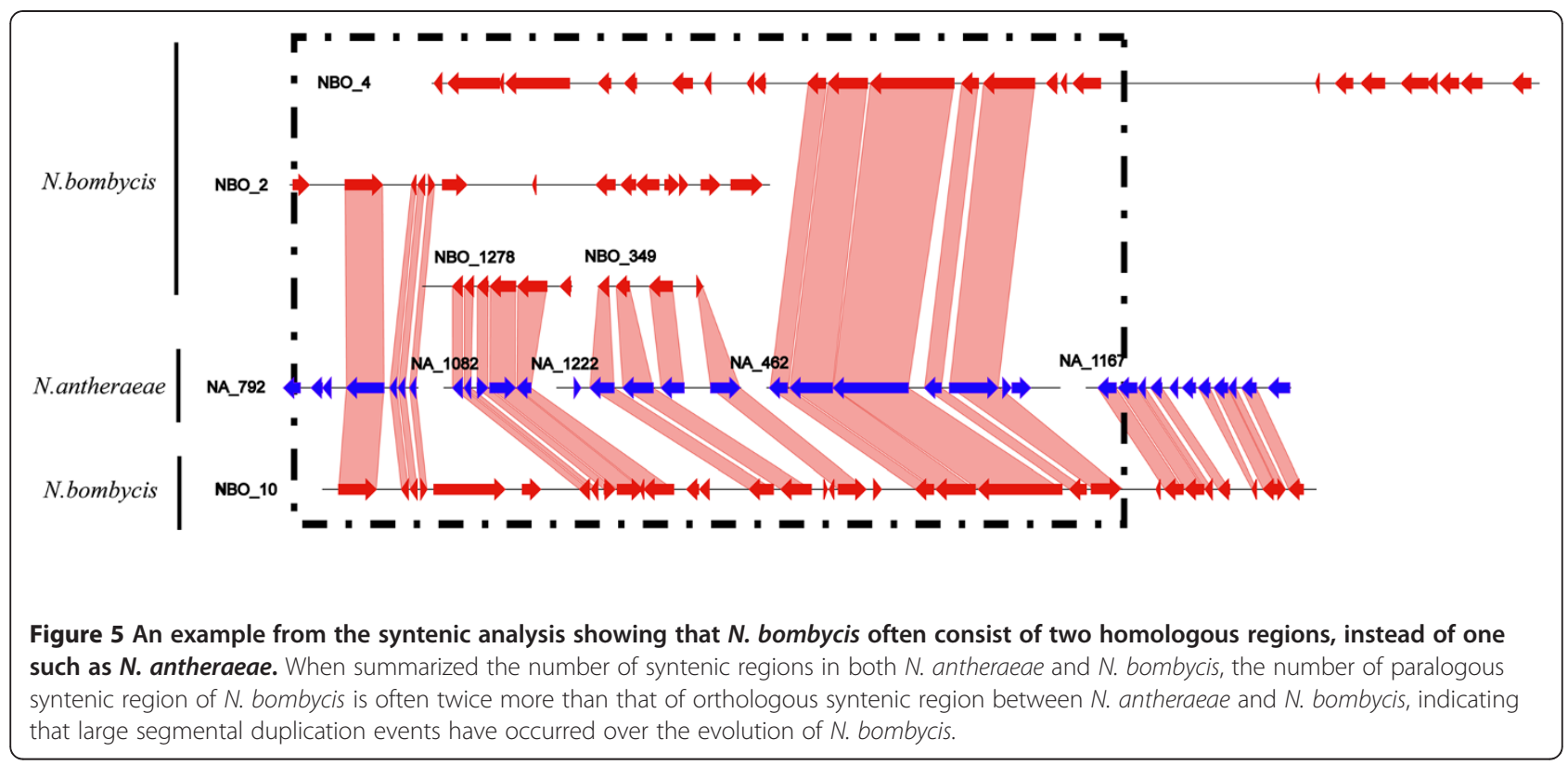

mori are up-regulated during the infection of $N$. bombycis (Figure 6). The upregulation of $\beta-G R P 2$ and $\beta$ GRP4 subsequently suppress the production of PPO against the infection of $N$. bombycis. To seek for the treatment method of pébrine, the interplay between SPN106 and $\beta$-GRP should be treated as our priority in the future studies. These observations lead us to hypothesize that adaptive evolution of serine protease inhibitor in $N$. bombycis might deter the melanization pathway by blocking the serine protease cascade in domesticated silkworms (Figure 6).

\section{Discussion and conclusion}

Preventing the infection of Nosema bombycis is one of the prime concerns in the domesticated silkworm industry. However, attempts to manage these pathogens have been hindered by our poor knowledge of the underlying molecular mechanisms contributing to the highly infectious ability of $N$. bombycis. In the absences of transformation, genetics, and axenic cultivation, the comparative genomics approach is one of the few tools available to tackle these issues. In this study, we compared the genome of the major commercial silkworm pathogen, $N$. bombycis, to those of $N$. antheraeae and $N$. ceranae. Our study showed that the large genome size in silkworm Nosema genome is due to the proliferation of host-derived transposable elements, horizontally transferred genes from prokaryotes, and the production of segmental and tandem duplicates. Previous studies on the characterization of microsporidian genomic architectures have focused more on the genome reduction aspect [15-19,34-36]. Although few studies assumed the possibility of genome expansion in the microsporidia [23,37], the direct evidence is lacking. From the genome streamlining perspective, it is evident that many metabolic essential genes (e.g., the tricarboxylic acid cycle, fatty acid $\beta$-oxidation, respiratory electron-transport chain always) are missing in the microsporidian genome. In stark contrast, our study provides the first solid evidence showing that the microsporidian genome can expand. Gene duplications and proliferation of host-derived transposable elements are the two predominant molecular mechanisms contributing to the genome expansion in $N$. bombycis.

Recently, two studies have reported that some genes in the microsporidia Encephalitozoon romaleae were derived from an ancestral host [38,39], but we did not find any evidence of host-derived genes in $N$. bombycis. Instead, some genes in the $N$. bombycis genome were apparently derived from prokaryotes or viruses by horizontal gene transfer, similar to other microsporidian genomes [26,27]. Surprisingly, these prokaryote-transferred genes could complement some important metabolic pathways in Nosema, indicative of its essentiality over the course of Nosema evolution. The mobility of transposable elements has been shown to be associated with the frequency of horizontal gene transfer $[40,41]$. Although the $N$. bombycis genome is composed of $\sim 38 \%$ repetivitive elements, only 55 genes were found to be horizontally transferred. Such observation indicated that a great number of transposons will not lead to higher rate of HGTs in $N$. bombycis. One explanation is that most transposons of $N$. bombycis have lost their activities such that the rate of HGTs is not enhanced. Alternatively, those repetitive elements do not possess the ability of capturing genes to facilitate the rate of HGTs.

The other major source of novel genetic materials in $N$. bombycis is the numerous paralogs by large-scale and small-scale duplication events. Some of them show 
Table 3 Site test of adaptive evolution for N. bombycis paralogous genes

\begin{tabular}{|c|c|c|c|c|c|c|c|}
\hline \multirow[t]{2}{*}{ Description } & \multirow[t]{2}{*}{ CPG } & \multirow[t]{2}{*}{ Members } & \multirow{2}{*}{$\begin{array}{l}\text { Duplicate } \\
\text { type }\end{array}$} & \multicolumn{3}{|c|}{ LRT statistics } & \multirow[t]{2}{*}{$\mathrm{dN} / \mathrm{dS}$} \\
\hline & & & & M1 vs. M2 & M7 vs. M8 & M8 vs. M8a & \\
\hline Hypothetical protein & CPG199 & 4 & $\mathrm{DD}$ & $17.30^{* * *}$ & $17.68^{* * *}$ & $17.07^{* * *}$ & 6.55472 \\
\hline Hypothetical protein & CPG293 & 4 & $\mathrm{SD}, \mathrm{DD}$ & $7.34^{*}$ & $7.58^{*}$ & $6.68^{*}$ & 67.43545 \\
\hline Hypothetical protein & CPG446 & 3 & $\mathrm{TD}$ & $15.34^{* * *}$ & $16.15^{* * *}$ & $16.52^{* * *}$ & 96.64162 \\
\hline Hypothetical protein & CPG767 & 3 & $\mathrm{TD}, \mathrm{DD}$ & $13.80^{* *}$ & $13.92^{* * *}$ & $13.26^{* *}$ & 12.30571 \\
\hline Surface adhesion protein & CPG844 & 3 & $S D, D D$ & $21.04^{* * *}$ & $21.03^{* * *}$ & $22.81^{* * *}$ & 6.72603 \\
\hline Serine protease inhibitor 106 & CPG945 & 3 & $\mathrm{TD}, \mathrm{DD}$ & 5.85 & 5.92 & 5.8 & 19.13308 \\
\hline Serine protease inhibitor 106 & CPG1974 & 2 & DD & $15.81^{* * *}$ & $16.31^{* *}$ & $15.81^{* * *}$ & 52.1588 \\
\hline LPXTG-motif cell wall anchor domain protein+ & CPG1776 & 2 & SD & $14.38^{* * *}$ & $14.39^{* *}$ & $14.38^{* * *}$ & 20.02006 \\
\hline DnaJ homolog subfamily C member 9 & CPG1792 & 2 & SD & $13.76^{* *}$ & $13.85^{* * *}$ & $19.87^{* * *}$ & 57.65414 \\
\hline NIK and IKK(beta) binding protein & CPG1878 & 2 & SD & $12.22^{* *}$ & $12.59^{* *}$ & $14.57^{* * *}$ & 36.70677 \\
\hline Replication-associated protein & CPG2140 & 2 & DD & $18.67^{* * *}$ & $18.76^{* * *}$ & $21.14^{* * *}$ & 15.15205 \\
\hline Glucan endo-1,6-beta-glucosidase & CPG1640 & 2 & DD & 5.72 & 5.72 & $9.64^{* *}$ & 61.53906 \\
\hline Integrator complex subunit 4 & CPG2120 & 2 & SD & $8.46^{*}$ & $8.59^{*}$ & $8.22^{*}$ & 14.58649 \\
\hline Hypothetical protein & CPG961 & 3 & DD & $21.04^{* * *}$ & $22.99^{* * *}$ & $19.99^{* * *}$ & 9.5982 \\
\hline Hypothetical protein & CPG1520 & 2 & SD & $15.28^{* * *}$ & $15.37^{* * *}$ & $15.13^{* * *}$ & 8.84271 \\
\hline Hypothetical protein & CPG1637 & 2 & SD & $18.44^{* * *}$ & $18.75^{* * *}$ & $20.57^{* * *}$ & 14.62068 \\
\hline Hypothetical protein & CPG987 & 3 & DD & $13.65^{* *}$ & $13.37^{* *}$ & $13.11^{* *}$ & 39.08442 \\
\hline Hypothetical protein & CPG1865 & 2 & SD & $10.90^{* *}$ & $10.94^{* *}$ & $10.70^{* *}$ & 23.75418 \\
\hline Hypothetical protein & CPG1967 & 2 & SD & $6.06^{*}$ & $6.81^{*}$ & 5.48 & 29.25563 \\
\hline Hypothetical protein & CPG2026 & 2 & DD & $8.28^{*}$ & $8.41^{*}$ & $15.98^{* * *}$ & 15.9724 \\
\hline Hypothetical protein & CPG2083 & 2 & DD & $29.01 * * *$ & $29.00 * * *$ & $31.16^{* * *}$ & 61.60344 \\
\hline hypothetical protein & CPG775 & 3 & SD & $14.05^{* * *}$ & $14.05^{* * *}$ & $12.37^{* *}$ & 5.3171 \\
\hline Hypothetical protein & CPG2128 & 2 & SD & $17.79^{* * *}$ & $17.85^{* * *}$ & $19.27^{* * *}$ & 45.49562 \\
\hline Hypothetical protein & CPG869 & 3 & SD, DD & $17.45^{* * *}$ & $17.85^{* * *}$ & $17.31^{* * *}$ & 8.16665 \\
\hline
\end{tabular}

Note: SD, segmental; TD, Tandem; DD, Disperse; M1 vs. M2, LRT statistic for model M1 versus M2; M7 vs. M8, LRT for model M7 versus M8; * Significance with $\mathrm{P}<0.05$; ${ }^{*}$ Significance with $\mathrm{P}<0.01$; ${ }^{* *}$ Significance with $\mathrm{P}<0.001$.

evidence of accelerated changes through the relaxation of purifying selection, whereas others show evidence of positive selection. In either case, these paralogs seem to have provided raw materials for functional innovations as we showed in this study. Among them, the serine protease inhibitor family stands out as potential targets to study the higher infectious rates in $N$. bombycis.

\section{Methods}

\section{Extraction of DNA, library construction, genome assembly, and annotation}

About $1 \times 10^{9}$ spores of $N$. bombycis CQ1, were isolated from infected silkworms in Chongqing. Using lysis buffer containing SDS and proteinase $\mathrm{K}$, the $N$. bombycis genomic DNA was extracted from the germinated spores for each library construction. $N$. antheraeae $Y Y$ isolate were collected from a farm in Henan of China and preserved in our lab, and then DNA was extracted with cetyl trimethylammonium bromide (CTAB) method. Detailed methods for extracting genomic DNAs can see the online supplementary materials (Additional file 16). After the library construction of plasmid and miniBAC, all sequence reads were obtained by Sanger and Illumina sequencing. To assemble the $N$. bombycis genome, the illumina reads were assembled by BGI's de novo assembly software [42], which assembled unique and frequency repeat areas of genome. Next, we assembled further from the mixed data of illumina scaffolds and Sanger reads by using Phrap program.

To ensure the quality of gene annotation, we enriched the ESTs data by constructing two cDNA phage libraries and two Illumina cDNA libraries. 11,155 high quality reads were obtained by Sanger sequencing and 307,900 reads were obtained by illumina sequencing. Finally, 1517 unique ESTs were obtained with average length of $430 \mathrm{bp}$. Next, $N$. bombycis protein-coding genes were annotated using the following three different software: (1) Glimmer (version 3.0) with low eukaryote parameters [43], (2) GeneMarkS (version 4.6) with low eukaryote parameters [44], and (3) Augustus (version 2.0) with default 


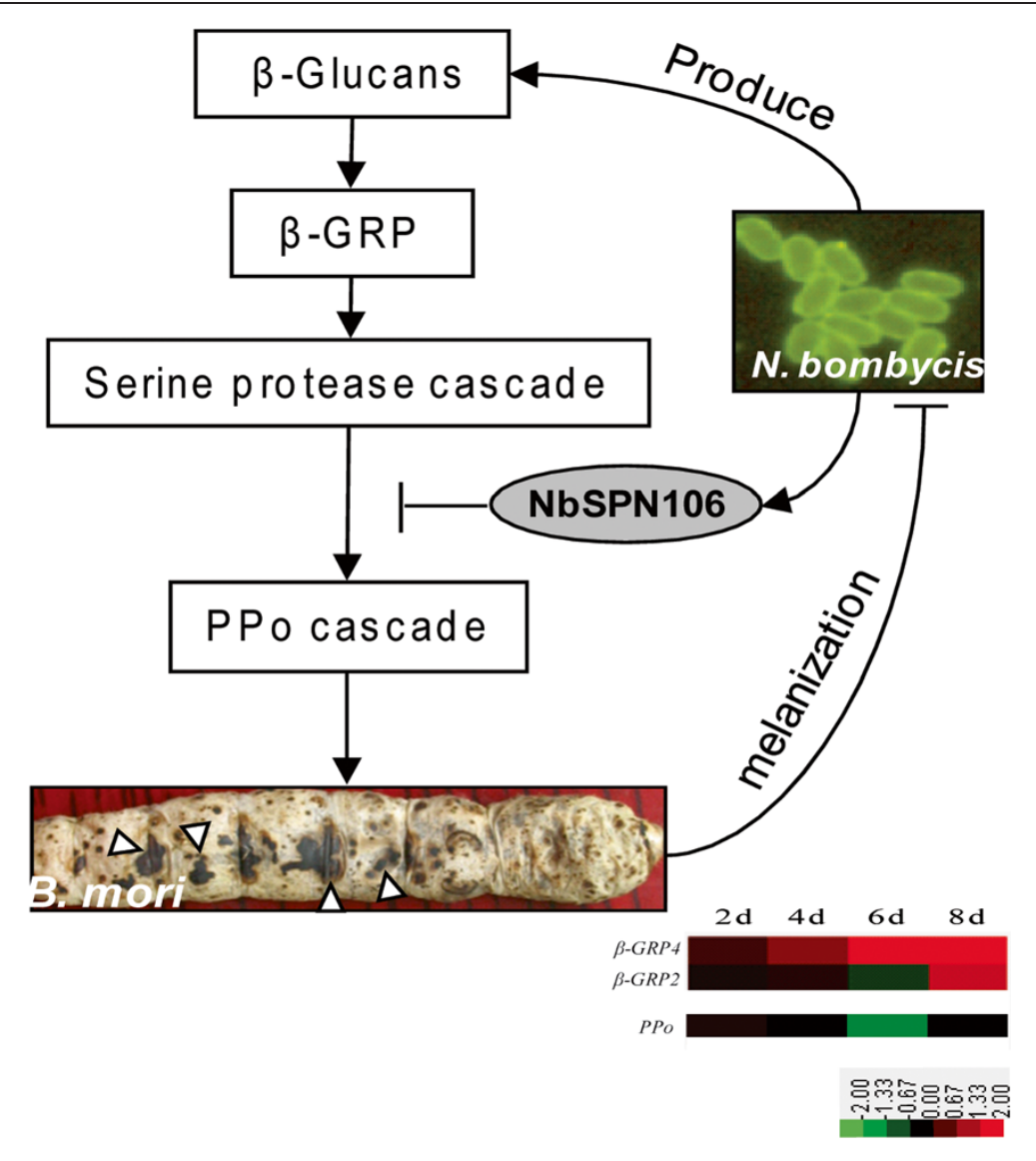

Figure 6 A hypothetical model showing how the SPN protein of $N$. bombycis suppresses the serine protease cascade of the melanization pathway of the host $\boldsymbol{B}$. mori. After the suppression of the serine protease cascade, the defensive response, the subsequent formation of melanization will be inhibited in the hosts. Abbreviation: PPO, prophenoloxidase; $\beta$-GRP, $\beta$-glucan recognition protein.

parameters [45]. The details of library construction of plasmid, miniBAC, DNA and cDNA, as well as the protocol of genome assembly and annotations, for $N$. bombycis and $N$. antheraeae are provided as online supplementary materials. All annotated sequences of $N$. bombycis and $N$. antheraeae are deposited in Genbank as the following accession numbers: ACJZ01000001-ACJZ01003558.

\section{Identification horizontal gene transfer (HGT)}

To examine the frequency of host-derived transposable elements, a phylogenetic analysis was conducted using the software RAxML [46] with the maximum likelihood (ML) algorithm. The amino acid replacement matrix, the WAG matrix, with gamma distribution was used to reconstruct the phylogenetic tree. Statistical support for nodes was estimated by using the bootstrapping method with $100 \mathrm{ML}$ replicates. All other HGT genes of the $N$. bombycis genome were identified by using both the phylogenetic method and the Darkhorse methods [47]. For the phylogenetic method, all initial $4,458 N$. bombycis genes were clustered to 3609 singletons at the level of $\geq 75 \%$ identity over $\geq 90 \%$ coverage for cluster members using BLASTCLUST program. A single randomly chosen representative of each cluster was used as a seed for BLASTP searches on $\mathrm{nr}$ database, the Bombyx mori genome database (http://silkworm.genomics.org.cn/). Sequences with E-value $<1$ e-5 and $>70 \%$ of the protein length) were aligned using clustal W. Bootstrap (100 replicates) consensus WAG model was made using RAxML to reconstruct Neighbor joining (NJ) trees. For the Darkhorse method, a filter threshold of $20 \%$ and two different self-definition keywords ( $N$. bombycis and all species name of Microsporidia phylum) were used to eliminate the BLASTP matches by calculating the lineage probability index (LPI) of genes in the $N$. bombycis genome. Then, the potential horizontally transferred genes were retrieved.

\section{Identification of segmental and tandem duplications}

To identify the segmental duplication, we performed allagainst-all blast search with a single species to identify 
collinear regions within single genome as segmental duplicated blocks. A collinear region was defined as one where there are at least three homologous pairs with $\mathrm{E}$ value $<1 \mathrm{E}-6$ and the distance between genes less than $5 \mathrm{~kb}$. Segmental blocks were visualized using the software Circos-0.55 [48]. To plot duplicated blocks among $N$. bombycis, $N$. antheraeae, and $N$. ceranae genomes, we ordered the scaffolds as follows: 1) only the scaffolds that shared syntenic genes among these three species were included; 2) the scaffolds of $N$. bombycis were ranked from longest to shortest; 3) scaffolds of the other two species were arranged based on synteny to $N$. bombycis; 4) if $N$. antheraeae or $N$. ceranae scaffolds were syntenic to more than two scaffolds of $N$. bombycis, we define that scaffold order based on the longest scaffold of $N$. bombycis.

For the identification of tandem duplicates, we first classified gene family using the software MCL with E value $<1 \mathrm{E}-10$, and then defined tandem duplicates as follows: 1 ) belonging to the same gene family, 2) being located within $5 \mathrm{~kb}$ each other, and 3) being separated by $\leq 3$ non-homologous genes.

To time the age of paralogs, we first identified collinear regions between $N$. bombycis and $N$. antheraeae. Then, genes that lie in the collinear region were classified as orthologs between $N$. bombycis and $N$. antheraeae. Synonymous substitution rate $(\mathrm{dS})$ of paralogs was estimated using the software Codeml in the package PAML 4 [49].

\section{Estimation of gene-wide selection and codon-based selection}

The gene-wide selection and codon-based selection of genes in $N$. bombycis were analyzed following the procedure described in [29]. Briefly, clusters of homologous genes in $N$. bombycis, $N$. antheraeae, and $N$. ceranae identified by MCL $(<1 \mathrm{E}-10)$ were grouped into four different categories: 1) clusters of orthologous genes (COGs) of 1:1:1 orthologous trios without any subsequent gene duplication in any species, 2) COGs of 1:1 N. bombycis and $N$. antheraeae gene pairs, 3) COGs of 1:1 N. bombycis and $N$. ceranae gene pairs, and 4) clusters of paralogous genes (CPGs) in $N$. bombycis. Prior to the estimation of $\mathrm{dN} / \mathrm{dS}$, those clusters with identity $<50 \%$ and an area covering $<50 \%$ of the length of each sequence were filtered. Multiple alignments using MUSCLE [50] were then parsed to remove those poorly aligned regions using the Gblocks algorithm [51] with the following criteria: maximum number of contiguous non-conserved positions $=10$ and minimum length of a block $=5$. The amino acid alignment was back-translated into nucleotides sequence alignment. The gene-wide selection was then determined by calculating the median $\mathrm{dN} / \mathrm{dS}$ value for each cluster. For the detection of codon-based positive selection, codon-based selection analysis was implemented using the Codeml program in the PAML package [49]. The site-specific model was used to detect positive selection in CPGs of $N$. bombycis. Two likelihood ratio tests were implemented: M1-M2 and M7-M8. M1 and M7 are the null models without positive selection, while M2 and M8 are the alternative models with positive selection. For each test, the first model (i.e., M1 or M7) is simpler than the second model (i.e., M2 or M8). To test if the second model fits better than the first model, twice difference of logarithm maximum likelihood estimates between the two compared models was compared against chi-square distribution with two degrees of freedom. Only those that showed posterior probability $>0.95$ in the empirical Bayes method were considered as positively selected sites [52,53].

\section{RNA labeling and hybridization}

RNA labeling and microarray hybridization was conducted by CapitalBio Corp (Beijing, China). Gene expression analysis was done based on the Affymetrix Silkworm Gene Chip kit in accordance following the manufacturer's instruction (http://www.capitalbio.com). Briefly, after $5 \times 10^{4}$ spores were fed to 3-instar larvae, total RNA was isolated from those 3-instar larvae at day 2, 4, 6, and 8. Then the extracted total RNA was reverse transcribed into cDNA. A dual-dye experiment was conducted. The uninfected cDNAs were labeled with dye Cy3 and infected cDNAs were labeled with dye Cy5. The labeled cDNA probes were dissolved in hybridization solution overnight at $42^{\circ} \mathrm{C}$ and then hybridized to the $23 \mathrm{k}$ silkworm genome oligonucleotide chip (Capital Bio) that consists of 22,987 oligonucleotide 70 -mer probes [54]. The signals were scanned with LuxScan 10KA scanner (CapitalBio corp).Three biological repeats were conducted at each time point.

\section{Additional files}

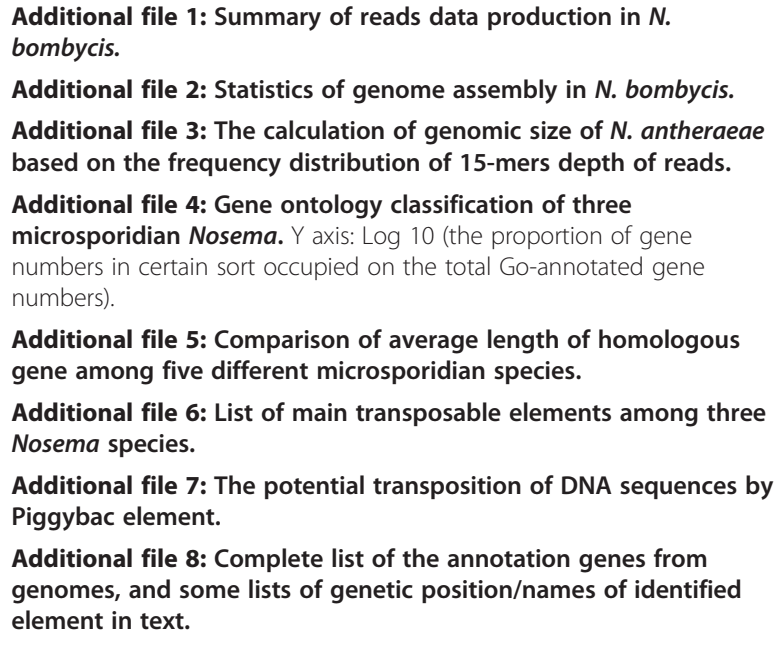

Additional file 8: Complete list of the annotation genes from genomes, and some lists of genetic position/names of identified element in text. 
Additional file 9: Diagram showing the Piggybac transposonmediated exogenous DNA sequence in the collinear region of $N$. bombycis. TTAA indicates the recognition site of the Piggybac transposon.

Additional file 10: Summary of 55 horizontally transferred genes in the $N$. bombycis genome.

Additional file 11: Figure showing phosphomevalonate kinase that horizontal transfer from bacteria integrates the mevalonate pathway of $N$. bombycis.

Additional file 12: The location of segmental duplications in Nosema bombycis genome.

Additional file 13: Cumulative plot and statistics of the $\mathrm{dN} / \mathrm{dS}$ values for CPG and COG genes in N. bombycis.

Additional file 14: The numbers of EST tags for positively selected CPG genes.

Additional file 15: Table of parameter estimates for all positively selected CPGs.

Additional file 16: Supplementary materials for library construction and genomic assembly.

\section{Competing interests}

The author(s) declare that we have no competing interests.

\section{Authors' contributions}

Conceived and designed the experiments: ZYZ ZHX QYX JW CL ZZ NJH; Performed the experiments: GQP JSX TL CFL HDL LY XZ ZLW XQD MLT YHL; Analyzed the data: GQP JSX TL SLL GJZ SGL TL WF HX; Contributed reagents /materials/analysis tools: JHH ZL LPL JL LNG LLW MXL YJW; Wrote the paper: GQP ZYZ JSX QYX SLL PJK. All authors read and approved the final manuscript.

\section{Acknowledgements}

We acknowledge the support of Prof. Christian P. Vivarès (Université Blaise Pascal, France) for his team's help on analysis of chromosomes organization of $N$. bombycis by PFGE. Thanks for the help of bioinformatics analysis from Chongqing NoeGen Bioinformatics Technology Co., LTD (http://www. noegen.com). This work is supported by the grants from National Basic Research Program of China (No.2012CB1 14604), Natural Science Foundation of China (No. 30930067, 31001037, 31001036, 31072089, 31272504, 31270138), National High-tech R\&D Program (863 Program, No. 2012AA101301-3, No. 2013AA102507), Chongqing Science \& Technology Commission (No. CSTC2010AA1003), the Program of Introducing Talents of Discipline to Universities (No.B07045), Key Project of Ministry of Education of China (No.210180).

The genome data and annotation information of N. bombycis and N. antheraeae were submitted to GenBank (Accession numbers ACJZ01000001 ACJZ01003558).

These data are also freely available at our public lab website: http://microbe. swu.edu.cn/bio/genome/nosema.

\section{Author details}

${ }^{1}$ State Key Laboratory of Silkworm Genome Biology, Southwest University, Chongqing 400715, China. ${ }^{2}$ College of Life Sciences, Chongqing Normal University, Chongqing 400047, China. ${ }^{3}$ Department of Botany, University of British Columbia, Vancouver, British Columbia V6T 1Z4, Canada. ${ }^{4}$ Beijing Genomics Institute at Shenzhen, Shenzhen 518000, China.

Received: 26 September 2012 Accepted: 26 February 2013 Published: 16 March 2013

\section{References}

1. Desportes I, Le Charpentier Y, Galian A, Bernard F, Cochand-Priollet B, Lavergne A, Ravisse P, Modigliani R: Occurrence of a new microsporidian: Enterocytozoon bieneusi n.g., n.sp., in the enterocytes of a human patient with AIDS. Journal of Protozool 1985, 32:250-254.

2. Snowden KF: Zoonotic microsporidia from animals and arthropods with a discussion of human infection. In Opporunistic infections: toxolasma, sarcocystis, and microsporida. Edited by Lindsay DS, Weiss LM. Boston, MA: World Class Parasites; 2004:123-134

3. Wittner M: Historic perspective on the microsporidia: expanding horizons. In The microsporidia and microsporidiosis. Edited by Wittner M, Weiss LM. Washington, DC: ASM Press; 1999:1-6.

4. Nageli K: Uber die neue krankheit der seidenraupe und verwandte organismen. Bot Z 1857, 15:760-761.

5. Pasteur L: Études sur la maladie des vers a soie: moyen pratique assurÉ de la combattre et d'en prÉvenir le retour. Paris: Imprimeur-Libraire; 1870.

6. Kashkarova L, Khakhanov A: Range of the hosts of the causative agent of pébrine (Nosema bombycis) in the mulberry silkworm. Parazitologiia 1980, $14: 164$.

7. Kudo R, DeCoursey J: Experimental infection of hyphantria cunea with nosema bombycis. J Parasitol 1940, 26:123-125

8. Hayasaka S, Yonemura N: Infection and development of Nosema sp. NIS H5 (Microsporida: Protozoa) in several lepidopteran insects. JARQ 1999, 33:65-68.

9. $\mathrm{Xu} J \mathrm{~S}$, Zhou ZY: Improving phylogenetic inference of microsporidian Nosema antheraeae among Nosema species with RPB1, $a$-and $\beta$-tubulin sequences. Afr J Biotechnol 2010, 9:7900-7904

10. Cornman RS, Chen YP, Schatz MC, Street C, Zhao Y, Desany B, Egholm M, Hutchison S, Pettis JS, Lipkin WI, Evans JD: Genomic analyses of the microsporidian Nosema ceranae, an emergent pathogen of honey bees. PLOS Pathog 2009, 5:e1000466.

11. Keeling PJ, Fast NM: Microsporidia: biology and evolution of highly reduced intracellular parasites. Annu Rev Microbiol 2002, 56:93-116.

12. Corradi $\mathrm{N}$, Slamovits $\mathrm{CH}$ : The intriguing nature of microsporidian genomes. Brief Funct Genomics 2011, 10:115-24.

13. Kawakami Y, Inoue T, Ito K, Kitamizu K, Hanawa C, Ando T, Iwano H, Ishihara $\mathrm{R}$ : Identification of a chromosome harboring the small subunit ribosomal RNA gene of Nosema bombycis. J Invertebr Pathol 1994, 64:147.

14. Xu J, Wang L, Tang F, Huang W, Zhou Z: The nuclear apparatus and chromosomal DNA of the microsporidian nosema antheraeae. J Eukaryot Microbiol 2011, 58:178-180.

15. Katinka MD, Duprat $S$, Cornillot E, Méténier G, Thomarat F, Prensier G, Barbe V, Peyretaillade E, Brottier P, Wincker P, Delbac F, El Alaoui H, Peyret P, Saurin W, Gouy M, Weissenbach J, Vivarès CP: Genome sequence and gene compaction of the eukaryote parasite Encephalitozoon cuniculi. Nature 2001, 414:450-453.

16. Slamovits CH, Fast NM, Law JS, Keeling PJ: Genome compaction and stability in microsporidian intracellular parasites. Curr Biol 2004, 14:891-896.

17. Akiyoshi DE, Morrison HG, Lei S, Feng X, Zhang Q, Corradi N, Mayanja H, Tumwine JK, Keeling PJ, Weiss LM, Tzipori S: Genomic survey of the noncultivatable opportunistic human pathogen. Enterocytozoon bieneusi. PLoS pathogens 2009, 5:e1000261.

18. Corradi N, Pombert JF, Farinelli L, Didier ES, Keeling PJ: The complete sequence of the smallest known nuclear genome from the microsporidian Encephalitozoon intestinalis. Nat Commun 2010, 1:77.

19. Keeling PJ, Corradi N, Morrison HG, Haag KL, Ebert D, Weiss LM, Akiyoshi DE, Tzipori S: The reduced genome of the parasitic microsporidian enterocytozoon bieneusi lacks genes for core carbon metabolism. Genome Biol Evol 2010, 2:304

20. Feschotte C, Jiang N, Wessler SR: Plant transposable elements where genetics meets genomics. Nat Rev Genet 2002, 3:329-41.

21. Hinkle G, Morrison H, Sogin M: Genes coding for reverse transcriptase, DNA-directed RNA polymerase, and chitin synthase from the microsporidian Spraguea lophii. Biol Bull 1997, 193:250.

22. Xu J, Pan G, Fang L, Li J, Tian X, Li T, Zhou Z, Xiang Z: The varying microsporidian genome: existence of long-terminal repeat retrotransposon in domesticated silkworm parasite Nosema bombycis. Int J Parasitol 2006, 36:1049-1056.

23. Williams BA, Lee RC, Becnel JJ, Weiss LM, Fast NM, Keeling PJ: Genome sequence surveys of Brachiola algerae and Edhazardia aedis reveal microsporidia with low gene densities. BMC Genomics 2008, 9:200.

24. Jinshan X, Jie L, Bettina D-V, Xiaoyan Z, Hangdeng L, Zeyang Z: Characterization of a transcriptionally active Tc1-like transposon in the microsporidian Nosema bombycis. Acta Parasitologica 2010, 55:8-15.

25. Xu J, Wang M, Zhang X, Tang F, Pan G, Zhou Z: Identification of NbME MITE families: potential molecular markers in the microsporidia nosema bombycis. J Invertebr Pathol 2010, 103:48-52. 
26. Fast NM, Law JS, Williams BAP, Keeling PJ: Bacterial catalase in the microsporidian Nosema locustae: implications for microsporidian metabolism and genome evolution. Eukaryot Cell 2003, 2:1069-1075.

27. Richards TA, Hirt RP, Williams B, Embley TM: Horizontal gene transfer and the evolution of parasitic protozoa. Protist 2003, 154:17.

28. Lynch M: The origins of genome architecture. Sinauer Press; 2007.

29. Emes RD, Yang Z: Duplicated paralogous genes subject to positive selection in the genome of Trypanosoma brucei. PLoS One 2008, 3:e2295.

30. Turner PC, Moyer RW: Poxvirus immune modulators: functional insights from animal models. Virus Res 2002, 88:35-53.

31. Richardson J, Viswanathan K, Lucas A: Serpins, the vasculature, and viral therapeutics. Front Biosci 2006, 11:1042-1056

32. Tao M, Pan G, Hu H, Zhou Z: :Identification of a new serpin gene (NbSPN106) from Nosema bombycis. Wei sheng wu xue bao = Acta Microbiol Sin 2009, 49:726.

33. Tanaka H, Yamakawa M: Regulation of the innate immune responses in the silkworm, Bombyx mori. ISJ 2011, 8:59-69.

34. Corradi N, Akiyoshi DE, Morrison HG, Feng X, Weiss LM, Tzipori S, Keeling PJ: Patterns of genome evolution among the microsporidian parasites encephalitozoon cuniculi. Antonospora locustae and Enterocytozoon bieneusi. Plos One 2007, 2:e1277.

35. Tsaousis AD, Kunji ER, Goldberg AV, Lucocq JM, Hirt RP, Embley TM: A novel route for ATP acquisition by the remnant mitochondria of Encephalitozoon cuniculi. Nature 2008, 453:553-556.

36. Gill E: Fast NS:tripped-down DNA repair in a highly reduced parasite. BMC Mol Biol 2007, 8:24.

37. Corradi N, Haag KL, Pombert JF, Ebert D, Keeling PJ: Draft genome sequence of the Daphnia pathogen Octosporea bayeri: insights into the gene content of a large microsporidian genome and a model for hostparasite interactions. Genome Biol 2009, 10:R106.

38. Selman M, Pombert JF, Solter L, Farinelli L, Weiss LM, Keeling P, Corradi N: Acquisition of an animal gene by microsporidian intracellular parasites. Curr Biol 2011, 21:R576.

39. Pombert JF, Selman M, Burki F, Bardell FT, Farinelli L, Solter LF, Whitman DW, Weiss LM, Corradi N, Keeling PJ: Gain and loss of multiple functionally related, horizontally transferred genes in the reduced genomes of two microsporidian parasites. Proc Natl Acad Sci USA 2012, 109:38-43.

40. Keeling PJ, Palmer JD: Horizontal gene transfer in eukaryotic evolution Nat Rev Genet 2008, 9:605-618.

41. Archibald J, Richards T: Gene transfer: anything goes in plant mitochondria. BMC Biol 2010, 8:147.

42. Li R, Zhu H, Ruan J, Qian W, Fang X, Shi Z, Li Y, Li S, Shan G, Kristiansen K, Li S, Yang H, Wang J, Wang J: De novo assembly of human genomes with massively parallel short read sequencing. Genome Res 2010, 20:265.

43. Delcher AL, Harmon D, Kasif S, White O, Salzberg SL: Improved microbial gene identification with GLIMMER. Nucleic Acids Res 1999, 27:4636.

44. Lomsadze A, Ter-Hovhannisyan V, Chernoff YO, Borodovsky M: Gene identification in novel eukaryotic genomes by self-training algorithm. Nucleic Acids Res 2005, 33:6494.

45. Stanke M, Diekhans M, Baertsch R, Haussler D: Using native and syntenically mapped cDNA alignments to improve de novo gene finding. Bioinformatics 2008, 24:637

46. Stamatakis A: RAxML-VI-HPC: maximum likelihood-based phylogenetic analyses with thousands of taxa and mixed models. Bioinformatics 2006, 22:2688

47. Podell S, Gaasterland T: DarkHorse: a method for genome-wide prediction of horizontal gene transfer. Genome Biol 2007, 8:R16.

48. Krzywinski M, Schein J, Birol I, Connors J, Gascoyne R, Horsman D, Jones SJ, Marra MA: Circos: an information aesthetic for comparative genomics. Genome Res 2009, 19:1639.

49. Yang Z: PAML4: phylogenetic analysis by maximum likelihood. Mol Biol Evol 2007, 24:1586-1591.

50. Edgar R: MUSCLE: a multiple sequence alignment method with reduced time and space complexity. BMC Bioinforma 2004, 5:113.

51. Castresana J: Selection of conserved blocks from multiple alignments for their use in phylogenetic analysis. Mol Biol Evol 2000, 17:540.
52. Nielsen R, Yang Z: Likelihood models for detecting positively selected amino acid sites and applications to the HIV-1 envelope gene. Genetics 1998, 148:929

53. Wong WSW, Yang Z, Goldman N, Nielsen R: Accuracy and power of statistical methods for detecting adaptive evolution in protein coding sequences and for identifying positively selected sites. Genetics 2004, 168:1041.

54. Huang L, Cheng T, Xu P, Cheng D, Fang T, Xia Q: A genome-wide survey for host response of silkworm. Bombyx mori during pathogen bacillus bombyseptieus infection. PloS one 2009, 4:e8098.

doi:10.1186/1471-2164-14-186

Cite this article as: Pan et al:: Comparative genomics of parasitic silkworm microsporidia reveal an association between genome expansion and host adaptation. BMC Genomics 2013 14:186.

\section{Submit your next manuscript to BioMed Central and take full advantage of:}

- Convenient online submission

- Thorough peer review

- No space constraints or color figure charges

- Immediate publication on acceptance

- Inclusion in PubMed, CAS, Scopus and Google Scholar

- Research which is freely available for redistribution

Submit your manuscript at www.biomedcentral.com/submit
C Biomed Central 